Artículo original

\section{Helicobacter pylori: resistencia múltiple en pacientes de Bogotá, Colombia}

\author{
Azucena Arévalo ${ }^{1,2}$, William Otero $^{3}$, Alba Alicia Trespalacios ${ }^{1}$ \\ ${ }^{1}$ Laboratorio de Bacteriología Especial, Grupo de Enfermedades Infecciosas, Departamento de \\ Microbiología, Facultad de Ciencias, Pontificia Universidad Javeriana, Bogotá, D.C., Colombia \\ ${ }^{2}$ Facultad de Medicina Veterinaria y Zootecnia, Universidad Nacional de Colombia, Bogotá, D.C., \\ Colombia \\ ${ }^{3}$ Unidad de Gastroenterología, Facultad de Medicina, Universidad Nacional de Colombia, \\ Bogotá, D.C., Colombia
}

Introducción. La resistencia a los antibióticos es la principal causa del fracaso del tratamiento contra Helicobacter pylori; la claritromicina y el metronidazol son los antibióticos que generan mayor resistencia. En Colombia, la resistencia primaria a estos dos antibióticos y el uso excesivo de levofloxacina han alcanzado los límites aceptados $(13,6,83$ y $16 \%$, respectivamente). A pesar de ello, se usa el tratamiento empírico combinando estos antibióticos en pacientes en los que ha fallado anteriormente.

Objetivo. Determinar la resistencia a los antibióticos en pacientes previamente tratados para $H$. pylori en Bogotá, Colombia.

Materiales y métodos. Se llevó a cabo un estudio descriptivo en el que se evaluó mediante dilución en agar la resistencia a la amoxicilina, la claritromicina, la levofloxacina y el metronidazol en 10 aislamientos provenientes de 5 pacientes con tres o cuatro tratamientos fallidos para $H$. pylori. La resistencia a los antibióticos se confirmó mediante secuenciación de ADN (Magrogen, Korea).

Resultados. Ocho de los aislamientos presentaron resistencia a dos o más antibióticos y todos fueron resistentes a la levofloxacina. Los patrones de sensibilidad de los aislamientos provenientes del antro pilórico y del cuerpo del estómago, fueron diferentes en tres de los pacientes.

Conclusión. Hasta donde se sabe, esta es la primera evidencia de resistencia múltiple de H. pylori en Colombia en pacientes previamente tratados. Los resultados evidenciaron las consecuencias del uso de un esquema ineficaz de tratamiento antibiótico y la necesidad de evaluar la sensibilidad a los antibióticos en diferentes sitios anatómicos del estómago. La resistencia múltiple limita el número de antibióticos útiles para erradicar $\mathrm{H}$. pylori.

Palabras clave: Helicobacter pylori; resistencia a múltiples medicamentos; antibacterianos; mutación; terapia.

Recibido: 05/04/18

Aceptado: $17 / 10 / 18$

Citación:

Arévalo A, Otero W, Trespalacios AA. Helicobacter pylori: resistencia múltiple en pacientes de Bogotá, Colombia. Biomédica. 2019;39(Supl.1):125-34 https://doi.org/10.7705/biomedica.v39i3.4437

\section{Correspondencia:}

William Otero, Calle $91 \mathrm{~N}^{\circ} 19 \mathrm{C}-62$, oficina 701, Bogotá, D.C., Colombia

Teléfono: (310) 7799823

waoteror@gmail.com

Contribución de los autores:

William Otero: diseño del estudio

Azucena Arévalo: realización de los experimentos, recolección y análisis de los datos

Alba Alicia Trespalacios: análisis de los datos

Todos los autores participaron en la escritura del manuscrito.

Financiación:

Este trabajo fue apoyado por la Pontificia Universidad Javeriana, subvención 00004554, en el marco del proyecto: "Efecto del polimorfismo CYP2C19 en la erradicación de Helicobacter pylori".

Conflicto de intereses:

Los autores declaran no tener conflicto de intereses.

\section{Helicobacter pylori: Multiple resistance in patients from Bogotá, Colombia}

Introduction: The main cause for Helicobacter pylori infection treatment failure is antibiotic resistance, where clarithromycin and metronidazole play the main role. In Colombia, primary resistance as a consequence of the use of these two antibiotics and excessive levofloxacin use is above the accepted limit (13.6\%, $83 \%$, and $16 \%$, respectively). Despite this fact, empirical therapies that include the combination of these antibiotics are used in patients with previous therapeutic failure.

Objective: To determine antibiotic resistance in patients previously treated for $H$. pylori in Bogotá, Colombia.

Materials and methods: We conducted a descriptive study that included ten isolates obtained from five patients with three or four previous failed treatments for $\mathrm{H}$. pylori. Antibiotic resistance to amoxicillin, clarithromycin, levofloxacin, and metronidazole was investigated by agar dilution and confirmed by DNA sequencing (Magrogen, Korea). Results: Eight isolates were resistant to two or more antibiotics. All isolates were resistant to levofloxacin. Susceptibility patterns in isolates from the gastric antrum and the body of the stomach were different in three patients.

Conclusion: As far as we know, this is the first evidence of multiple $H$. pylori resistance in Colombia in previously treated patients. Results demonstrated the consequences of using an ineffective antibiotic scheme and the need to assess antibiotic susceptibility in different anatomical sites of the stomach. The consequences of multiple resistance decrease possible antibiotic effectiveness to eradicate $H$. pylori in the future.

Keywords: Helicobacter pylori; drug resistance, multiple; anti-bacterial agents; susceptibility test; mutation; therapy. 
Helicobacter pylori es una bacteria microaerófila Gram negativa capaz de colonizar la mucosa gástrica; causa gastritis crónica en el $100 \%$ de las personas infectadas, úlceras gástricas en 10 a $20 \%$, linfoma de tejido linfoide asociado con la mucosa gástrica (Mucosa-Associated Lymphoid Tissue, MALT) en menos del $1 \%$ y adenocarcinoma gástrico distal en 1 a $2 \%$.

Helicobacter pylori es el principal factor de riesgo para el desarrollo de cáncer gástrico y ha sido clasificado como carcinógeno de tipo I por la Organización Mundial de la Salud (OMS) $(1,2)$, por lo cual, los expertos mundiales recomiendan que debe erradicarse siempre $(3,4)$. Sin embargo, a nivel mundial existe preocupación porque la eficacia de los tratamientos de primera línea comúnmente empleados es inferior al $80 \%$ (5), lo cual también ocurre en Colombia, como quedó demostrado en un estudio de Trespalacios, et al. (Trespalacios S, Otero W, Mercado M. Efficacy of firstline Helicobacter pylori eradication treatment with two triple regimens with levofloxacin. Gastroenterology. 2014;S-741-2. https://doi.org/10.1016/S00165085(14)62685-8).

Si bien el éxito terapéutico responde a múltiples factores ambientales o relativos al huésped, como el incumplimiento del tratamiento, y a la presencia de polimorfismos en la interleucina $1 \mathrm{~B}$ (IL-1B) en enzimas involucradas en el metabolismo de los medicamentos (CYP2C19 y CYP3A4) y en el de las bacterias, se ha demostrado que la principal causa del fracaso del tratamiento es la resistencia bacteriana (6). Por ello, en febrero de 2017, la OMS incluyó a $H$. pylori en la lista de los 16 microorganismos que amenazan a la humanidad y ameritan urgentes medidas de control, clasificándolo en la categoría de más alta prioridad, debido al aumento de la resistencia a los antibióticos de primera línea, metronidazol y claritromicina, especialmente a este último (7), y a la aparición de aislamientos multirresistentes.

A nivel mundial, hay pocos estudios sobre la multirresistencia de $H$. pylori, es decir, la resistencia a tres o más antibióticos, pero se estima que aproximadamente $15 \%$ de los aislamientos clínicos la presentan; esto implica una disminución de la tasa de erradicación y, por lo tanto, una grave amenaza para la salud humana (8). En el caso de H. pylori, la multirresistencia se da simultáneamente frente a amoxicilina, metronidazol y claritromicina $(9,10)$.

En general, $H$. pylori se hace resistente a amoxicilina, claritromicina, metronidazol, levofloxacina y tetraciclina, principalmente, mediante mutaciones puntuales en un par de bases del gen que codifica para el sitio de acción del antibiótico, o por acción de las bombas de expulsión bacterianas, que normalmente protegen al microorganismo de la toxicidad de los antibióticos (11-18). Las bombas de expulsión son un complejo estructural de proteínas en la membrana interna de las bacterias, que actúa en sincronía con proteínas adaptadoras del periplasma y canales presentes en la membrana externa, las cuales expulsan eficientemente los medicamentos (19). Dichas proteínas se asocian, especialmente, con el desarrollo de multirresistencia, como en el caso de la codificada por el gen hefA $(\mathrm{p}=0,003)(20)$.

En Colombia, la resistencia primaria a claritromicina, metronidazol y levofloxacina es alta, de 13,6, 81 y $27 \%$, respectivamente $(14,21,22)$; esto limita su utilización en los tratamientos empíricos de primera línea. Sin embargo, son escasos los estudios en pacientes en quienes ya ha fracasado el tratamiento, pues, a menudo, se excluyen de los estudios de 
sensibilidad (23). Además, el número cada vez mayor de tratamientos de erradicación puede haber resultado en que no se investiguen más pacientes con infecciones resistentes al tratamiento (23). Sin embargo, la vigilancia de la presencia de aislamientos de $\mathrm{H}$. pylori multirresistente es esencial para permitir el uso racional de los antibióticos después de repetidos fracasos terapéuticos, especialmente en lugares donde no se dispone de las pruebas de sensibilidad a los antibióticos (24).

En este contexto, el objetivo de este estudio fue analizar los aislamientos clínicos $H$. pylori de pacientes con varias fallas terapéuticas previas en Bogotá, Colombia.

\section{Materiales y métodos}

\section{Tipo de estudio y pacientes}

Se llevó a cabo un estudio descriptivo en el que se incluyeron 5 pacientes que fueron previamente excluidos de un ensayo clínico de 355 pacientes (26) debido a que habían recibido tratamiento previo para $H$. pylori. A los 5 pacientes incluidos en este estudio se les realizó endoscopia de vías digestivas altas en diferentes centros de Bogotá entre febrero y noviembre de 2014. Los motivos de consulta fueron dispepsia y reflujo en cinco pacientes, así como una historia de infección por $H$. pylori de difícil tratamiento, de 2 a 14 años de evolución.

Después de la explicación médica sobre el procedimiento endoscópico y sobre el estudio, los pacientes firmaron el consentimiento informado. Posteriormente, se hizo la endoscopia de vías digestivas altas después de un ayuno mínimo de seis horas (21). Se tomaron biopsias de rutina para el estudio de la gastritis crónica, según el protocolo propuesto por Rugge, et al. (25), y biopsias adicionales para el cultivo de H. pylori: cuatro biopsias del antro y cuatro del cuerpo del estómago. Una de las biopsias de antro se sometió a la prueba rápida de ureasa.

\section{Transporte de muestras y cultivo}

Las biopsias del antro y el cuerpo gástricos destinadas al cultivo de $H$. pylori, se colocaron en $500 \mu \mathrm{l}$ de caldo Brucella (Beckton Dickenson) con suplemento de $20 \%$ (p/v) de glicerol (Invitrogen) y se almacenaron a 4 ${ }^{\circ} \mathrm{C}$ hasta su procesamiento. Los aislamientos de $H$. pylori obtenidos y las biopsias restantes, se mantuvieron a $-70{ }^{\circ} \mathrm{C}(21)$.

Las biopsias se maceraron en condiciones asépticas y estériles, con un aplicador de madera previamente tratado en solución de carbón activado al $1 \%(\mathrm{p} / \mathrm{v})$, hasta que se logró la homogeneidad total (21). Posteriormente, se sembraron en agar Brucella (Beckton Dickenson) con suplemento de IsoVitaleX ${ }^{\mathrm{TM}}$ al $4 \%(\mathrm{v} / \mathrm{v}), 7 \%(\mathrm{v} / \mathrm{v})$ de sangre de caballo y $2 \mathrm{ml}$ de suplemento selectivo para H. pylori, DENTTM (Thermo Scientific Oxoid, UK).

Los cultivos se incubaron a $37^{\circ} \mathrm{C}$ en condiciones microaerofílicas durante 3 a 14 días. Después de la incubación, se observaron pequeñas colonias transparentes que se sometieron a identificación fenotípica mediante coloración de Gram (bacilos curvos Gram negativos), oxidasa (positiva), catalasa (positiva) y prueba rápida de ureasa (positiva) (21). Posteriormente, se obtuvieron cultivos puros, para lo cual se aislaron las colonias con las características apropiadas en agar Brucella y se incubaron a $37^{\circ} \mathrm{C}$ en condiciones de microaerofilia durante tres a cinco días. 


\section{Pruebas de sensibilidad fenotípica y genotípica}

La sensibilidad fenotípica a $H$. pylori se evaluó a partir de cultivos puros para amoxicilina, claritromicina, levofloxacina y metronidazol mediante la prueba de dilución en agar, según las recomendaciones de los Centers for Disease Control and Prevention (CDC) $(26,27)$. Los puntos de corte utilizados para determinar la resistencia fueron $\geq 1 \mu \mathrm{g} / \mathrm{ml}$ para amoxicilina, claritromicina y levofloxacina, y $8 \mu \mathrm{g} / \mathrm{ml}$ para metronidazol (27-31). Como control se usó la cepa de H. pylori NCTC 11637.

Además, los aislamientos se analizaron genotípicamente para identificar posibles mutaciones asociadas con la resistencia y confirmar la sensibilidad fenotípica. Se usó DNAzol ${ }^{\mathrm{TM}}$ (Invitrogen) para extraer el ADN genómico de las colonias de H. pylori aisladas del antro y del cuerpo del estómago.

Posteriormente, se amplificaron los genes bacterianos relacionados con la resistencia a la amoxicilina (pbp1A), la claritromicina (rna23s), la levofloxacina $(g y r A)$ y el metronidazol $(r d x A)$, mediante reacción en cadena de la polimerasa (PCR) convencional, según protocolos previamente estandarizados $(14,22)$ (cuadro 1).

Cuadro 1. Genes analizados y secuencia de los iniciadores utilizados identificación de M. bovis, Colombia, 2015

\begin{tabular}{lclcc}
\hline Antibiótico & Gen & Iniciadores & $\begin{array}{c}\text { Tamaño } \\
\text { (bp) }\end{array}$ & Referencia \\
\hline Amoxicilina & pbp1A & $\begin{array}{l}\text { F: GCT ATT CCA CGA CTT CTA AA } \\
\text { R: GCA AGG TTA CAA GCC CTA AA }\end{array}$ & 380 & $(12)$ \\
Claritromicina & $23 S$ rDNA & $\begin{array}{l}\text { F: CCA CAG CGA TGT GGT CTC AG } \\
\text { R: CTC CAT AAG AGC CAA AGC CC }\end{array}$ & 425 & $(32)$ \\
Levofloxacina & $g y r A$ & $\begin{array}{l}\text { F: TTT RGC TTA TTC MAT GAG CGT } \\
\text { R: RGCA GAC GGC TTG GTA RAA TA }\end{array}$ & 428 & $(33)$ \\
Metronidazol & $r d x A$ & $\begin{array}{l}\text { F: GACAATTATTAACGAGCGC } \\
\text { R: CCTCCAATAATGCAACTATC }\end{array}$ & 460 & $(15)$ \\
\hline
\end{tabular}

a Iniciadores utilizados para la PCR convencional con el tamaño del producto amplificado en pares de bases (bp)

\section{Análisis bioinformático}

La sensibilidad genotípica y las mutaciones relacionadas con la resistencia a los antibióticos, se determinaron con la ayuda de análisis bioinformáticos. Las mutaciones asociadas con la resistencia a la claritromicina se detectaron con la herramienta bioinformática BLAST-N (Basic Local Alignment Search Tool-N). Para analizar las secuencias de detección de mutaciones relacionadas con la resistencia a amoxicilina, levofloxacina y metronidazol se utilizó la BLAST-X. Se empleó la secuencia de referencia proveniente de $H$. pylori 26695 (taxid: 85962), número de acceso a GenBank CP003904.1.

\section{Estadística}

Los resultados se analizaron mediante estadística descriptiva.

\section{Resultados}

\section{Distribución de los pacientes}

Durante el 2014, 5 de los pacientes remitidos a endoscopia de vías digestivas habían recibido varios tratamientos contra $H$. pylori sin éxito: cuatro 
hombres y una mujer entre los 41 y los 57 años de edad. Todos informaron tener antecedentes familiares de enfermedades gástricas. Cuatro (4/5) refirieron tener antecedentes familiares de gastritis y, uno (1/5), antecedentes de úlcera péptica. Un paciente (1/5) refirió antecedentes familiares de cáncer gástrico y, tres (3/5), de otros tipos de tumores gastrointestinales, como cáncer de esófago, cáncer de páncreas y linfoma MALT (cuadro 2).

Cuadro 2. Distribución, principales características e historia familiar de los pacientes

\begin{tabular}{|c|c|c|c|c|c|c|c|c|c|}
\hline \multirow[b]{2}{*}{ No. } & \multirow[b]{2}{*}{ Sexo } & \multirow{2}{*}{$\begin{array}{l}\text { Edad } \\
\text { (años) }\end{array}$} & \multirow[b]{2}{*}{ IMC } & \multirow{2}{*}{$\begin{array}{l}\text { Número de } \\
\text { tratamientos }\end{array}$} & \multirow[b]{2}{*}{ Motivo de consulta } & \multirow{2}{*}{$\begin{array}{l}\text { Evolución } \\
\text { (años) }\end{array}$} & \multicolumn{3}{|c|}{ Historia familiar } \\
\hline & & & & & & & $\begin{array}{l}\text { Enfermedad } \\
\text { gástrica }\end{array}$ & $\begin{array}{l}\text { Cáncer } \\
\text { gástrico }\end{array}$ & $\begin{array}{l}\text { Otros } \\
\text { cánceres }\end{array}$ \\
\hline 1 & Masculino & 45 & 29,0 & 4 & $\begin{array}{l}\text { H. pylori difícil de tratar, } \\
\text { dispepsia y ERGE }\end{array}$ & 14 & Gastritis & No & Esófago \\
\hline 2 & Femenino & 51 & 26,7 & 4 & $\begin{array}{l}\text { H. pylori difícil de tratar, } \\
\text { dispepsia y ERGE }\end{array}$ & 14 & Gastritis & No & No \\
\hline 3 & Masculino & 42 & 24,6 & 3 & $\begin{array}{l}\text { H. pylori difícil de tratar, } \\
\text { dispepsia y ERGE }\end{array}$ & 10 & Gastritis & No & Linfoma MALT \\
\hline 4 & Masculino & 57 & 24,2 & 3 & $\begin{array}{c}\text { H. pylori difícil de tratar } \\
\text { y úlcera }\end{array}$ & SD & Úlcera péptica & Sí & No \\
\hline 5 & Masculino & 41 & 25,4 & 4 & $\begin{array}{l}\text { H. pylori difícil de tratar, } \\
\text { dispepsia y ERGE }\end{array}$ & 2 & Gastritis & No & Páncreas \\
\hline
\end{tabular}

ERGE: reflujo gastroesofágico; IMC: índice de masa corporal; SD: sin dato

\section{Pruebas de sensibilidad fenotípica y genotípica}

Todos los aislamientos de los pacientes tuvieron resultados positivos en la prueba rápida de ureasa. Se obtuvieron diez aislamientos de antro y de cuerpo del estómago (cinco de antro y cinco de cuerpo). A todos los aislamientos se les hizo antibiograma para amoxicilina, claritromicina, levofloxacina y metronidazol, mediante dilución en agar.

Según el análisis de sensibilidad fenotípica, en un solo paciente se obtuvieron dos aislamientos de $H$. pylori (uno del cuerpo del estómago y otro del antro) resistentes a la amoxicilina. Los aislamientos de tres de los pacientes fueron resistentes a la claritromicina (tres del cuerpo y dos del antro); en cuatro de los pacientes, se encontraron aislamientos resistentes al metronidazol (cuatro del cuerpo y tres del antro). Por último, los aislamientos de todos los pacientes fueron resistentes a la levofloxacina (cuadro 3).

Cuadro 3. Sensibilidad a los antibióticos

\begin{tabular}{|c|c|c|c|c|c|c|c|c|c|c|c|c|c|c|c|c|}
\hline \multirow{2}{*}{$\begin{array}{l}\text { Antibiótico } \\
\text { Sitio } \\
\text { anatómico } \\
\text { del estómago }\end{array}$} & \multicolumn{4}{|c|}{ Amoxicilina } & \multicolumn{4}{|c|}{ Claritromicina } & \multicolumn{4}{|c|}{ Levofloxacina } & \multicolumn{4}{|c|}{ Metronidazol } \\
\hline & \multicolumn{2}{|c|}{ Antro } & \multicolumn{2}{|c|}{ Cuerpo } & \multicolumn{2}{|c|}{ Antro } & \multicolumn{2}{|c|}{ Cuerpo } & \multicolumn{2}{|c|}{ Antro } & \multicolumn{2}{|c|}{ Cuerpo } & \multicolumn{2}{|c|}{ Antro } & \multicolumn{2}{|c|}{ Cuerpo } \\
\hline Sensibilidad & $\underset{(\mu \mathrm{g} / \mathrm{ml})}{\mathrm{CIM}}$ & $\begin{array}{c}\mathbf{S G} \\
(\operatorname{pbp} 1 \mathrm{~A})\end{array}$ & $\underset{(\mu \mathrm{g} / \mathrm{ml})}{\mathrm{CIM}}$ & $\begin{array}{c}\text { SG } \\
(\mathrm{pbp} 1 \mathrm{~A})\end{array}$ & $\underset{(\mu \mathrm{g} / \mathrm{ml})}{\mathrm{CIM}}$ & $\begin{array}{c}\text { SG } \\
\text { (rna23S) }\end{array}$ & $\underset{(\mu \mathrm{g} / \mathrm{ml})}{\mathrm{CIM}}$ & $\begin{array}{c}\text { SG } \\
(\text { rna23S) }\end{array}$ & $\underset{(\mu \mathrm{g} / \mathrm{ml})}{\mathrm{CIM}}$ & $\underset{(\operatorname{gyrA})}{\text { SG }}$ & $\underset{(\mu \mathrm{g} / \mathrm{ml})}{\mathrm{CIM}}$ & $\begin{array}{c}\text { SG } \\
(\text { gyrA) }\end{array}$ & $\underset{(\mu \mathrm{g} / \mathrm{ml})}{\mathrm{CIM}}$ & $\begin{array}{c}\text { SG } \\
(\mathrm{rdxA})\end{array}$ & $\underset{(\mu \mathrm{g} / \mathrm{ml})}{\mathrm{CIM}}$ & $\underset{(\mathrm{rdxA})}{\mathbf{S G}}$ \\
\hline Paciente 1 & 0,062 & TS & 0,25 & TS & 0,016 & TS & 0,125 & TS & 64 & N87I & 32 & N87I & 1 & SD & 16 & SD \\
\hline 응 Paciente 2 & 0,125 & TS & 0,125 & TS & 0,125 & TS & 64 & SD & 8 & N87K & 2 & N87K & 8 & SD & 16 & SD \\
\hline$\frac{\pi}{5}$ Paciente 3 & 0,016 & TS & 0,062 & TS & 0,125 & TS & 0,5 & TS & 1 & N87I & 4 & N87I & 1 & SD & 0,5 & SD \\
\hline ๘ Paciente 4 & 0,25 & SD & 0,25 & SD & 8 & A2143G & 32 & A2143G & 2 & SD & 4 & SD & 4 & TS & 64 & TS \\
\hline Paciente 5 & 1 & E406V & 1 & SD & 64 & A2143G & 64 & A2143G & 4 & D91N & 4 & D91N & 16 & R90K & 16 & R90K \\
\hline
\end{tabular}

CIM: concentración inhibitoria mínima; SG: sensibilidad genotípica: TS: tipo silvestre; SD: sin datos 
Se estudiaron las mutaciones asociadas con resistencia a amoxicilina, claritromicina, levofloxacina y metronidazol, determinando su presencia en los genes pbp1A, rna23s y gyrA, aunque en algunos aislamientos no fue posible analizar las secuencias debido a su mala calidad (cuadro 3).

Las mutaciones relacionadas con resistencia a los antibióticos evaluados, fueron la E406V para la amoxicilina y la A2143G para la claritromicina. Para el metronidazol, se detectó la mutación R90K en la proteína rdxA y, para la levofloxacina, se detectaron tres cambios diferentes en la proteína gyrA en las posiciones 87 y 91, así: N87I, N87K y D91N (cuadro 3).

Se detectó resistencia múltiple en ocho de los diez aislamientos. Dos de ellos (uno de antro y otro de cuerpo provenientes del mismo paciente) presentaron resistencia a dos antibióticos: levofloxacina y metronidazol. Cuatro aislamientos de antro y cuerpo de dos pacientes diferentes, fueron resistentes a tres antibióticos: claritromicina, levofloxacina y metronidazol. Dos aislamientos de otro paciente presentaron resistencia a los cuatro antibióticos: amoxicilina, claritromicina, levofloxacina y metronidazol (cuadro 3 ). Además, se detectaron diferencias entre los aislamientos de antro y de cuerpo, en los patrones de resistencia en cuatro pacientes, lo que indicaría una infección concomitante con diferentes cepas de H. pylori (cuadro 3).

\section{Discusión}

Estos resultados evidencian que en Bogotá se registraron aislamientos de $H$. pylori con resistencia a múltiples antibióticos después de tres años (20122015) de vigilancia. La aparición de multirresistencia podría ser la consecuencia de tratamientos previos fallidos, o de resistencia 'secundaria' a claritromicina, metronidazol y levofloxacina debida al uso extensivo de estos antibióticos en el tratamiento de enfermedades respiratorias, parasitarias o ginecológicas, y de infecciones urinarias, respectivamente $(34,35)$. Estos hallazgos implicarían que dichos antibióticos no deberían utilizarse empíricamente en los tratamientos de primera línea, por lo menos en Bogotá. Resultados similares se han registrado en otros países como Alemania, Bulgaria, Polonia, Brasil, Israel, China y Corea $(23,24,36-38)$, en donde se encontraron resistencias secundarias múltiples a claritromicina, metronidazol y levofloxacina, con una mayor prevalencia de resistencia a claritromicina y metronidazol.

Los aislamientos del cuerpo y del antro presentaron diferentes patrones de sensibilidad, lo cual también se ha reportado previamente en Corea, China y Alemania (39-41). Este hallazgo explicaría el fracaso del tratamiento y no solo indicaría la presencia de más de una cepa de $H$. pylori, sino que subrayaría la importancia metodológica de hacer la prueba de sensibilidad a partir de muestras de ambos segmentos del estómago, con el fin de orientar mejor la elección del tratamiento y evitar recaídas de infección por $H$. pylori.

Los resultados del presente estudio resaltan la importancia de implementar pruebas de sensibilidad, ya sea mediante cultivo o biología molecular, antes de un primer tratamiento para $\mathrm{H}$. pylori, con el fin de orientar las terapias de primera línea, lo que concuerda con lo reportado por Park, et al. (38), en cuyo estudio se evidencia que, cuando el tratamiento de primera línea se administra teniendo en cuenta la sensibilidad a los antibióticos, la eficacia es superior a la del tratamiento empírico. Con la prueba previa de sensibilidad, la eficacia fue de $94,7 \%\left(\mathrm{IC}_{95 \%} 88,8-100\right)$ y por protocolo fue de $96,4 \%\left(\mathrm{IC}_{95 \%}\right.$ $91,5-100)$ y, con el tratamiento empírico fueron de $71,9 \%\left(\mathrm{IC}_{95 \%} 60,2-83,5\right)$ y $73,2 \%\left(\mathrm{IC}_{95 \%} 61,5-84,8\right)$, respectivamente (38). No obstante, cuando ya 
han fallado varios tratamientos, la utilidad del cultivo no es la misma debido a la aparición de resistencia, lo cual se suma a que el cultivo y las pruebas moleculares no están disponibles en todas partes y, por lo tanto, lo usual es recurrir a tratamientos empíricos (42), caso en que debería tenerse en cuenta la epidemiología de la resistencia local.

En Colombia, el tratamiento triple de primera línea se utilizó en el pasado, combinando amoxicilina, claritromicina o metronidazol o levofloxacina (43). Sin embargo, en otros estudios, se ha demostrado frecuentemente resistencia primaria a estos antibióticos (22), por lo cual ya no se recomiendan como una primera opción en el país (43).

El tratamiento empírico con estos antibióticos, sin tener en cuenta la epidemiologia local, con frecuencia conduce al fracaso terapéutico (38). Además, cuando hay resistencia a la claritromicina y al metronidazol, los tratamientos híbridos y concomitantes con esos antibióticos fracasan frecuentemente (35). En estos casos, la eficacia de los tratamientos triple, secuencial y concomitante, es de $\sim 50 \%,<20 \%$ y $50 \%$, respectivamente (44). En el informe del consenso Maastricht V/Florence (Maastricht V/Florence Consensus Report) se recomienda no usar tratamientos secuenciales ni concomitantes, sino cuádruples durante 14 días, en casos de resistencia simultánea a la claritromicina y el metronidazol superior al $15 \%(4,43)$; entre estos, están el que combina bismuto y furazolidona, más metronidazol o amoxicilina-tetraciclina, o los tratamientos con rifabutina (tratamiento de rescate), que también son una alternativa (45). La combinación de amoxicilina y tetraciclina tiene, generalmente, muy baja tasa de éxito: 62 a $75 \%$ por intención de tratar y 64 a $80 \%$ por protocolo.

Su escaso éxito podría deberse a la interacción del efecto bacteriostático de la tetraciclina con el efecto bactericida de la amoxicilina (45). La acción bactericida de la amoxicilina implica la inhibición de la formación de la pared celular, que depende de la tasa de proliferación de las bacterias (45). Los antibióticos bacteriostáticos, como la tetraciclina, pueden reducir la efectividad de la penicilina mediante la inhibición de la síntesis de proteínas celulares requerida para la división celular (45).

Se han explorado nuevas opciones terapéuticas para los casos de multirresistencia, entre ellas, el linezolid (46) y los nuevos análogos de las oxazolidionas (47). No obstante, el inconveniente es que el linezolid podría inducir la aparición de Staphylococcus spp. resistentes a su acción, por lo que la decisión sobre su uso requiere de más estudios $(46,47)$.

Los resultados del presente estudio evidenciaron la presencia de H. pylori con resistencia a múltiples antibióticos utilizados en los tratamientos empíricos, principalmente a claritromicina, levofloxacina y metronidazol, en ocho de diez aislamientos. Las pruebas de sensibilidad a los antibióticos deben hacerse, preferiblemente, antes de administrar el primer tratamiento para $\mathrm{H}$. pylori, en cuyo caso se sugiere hacerlas con muestras provenientes del antro y del cuerpo del estómago, para evitar que se pierdan aislamientos resistentes a los antibióticos en ambos sitios. Cuando hay $H$. pylori con resistencia a múltiples antibióticos, las opciones terapéuticas se restringen a los tratamientos cuádruples de rescate con furazolidona o rifabutina.

\section{Agradecimientos}

Agradecemos a los pacientes por su interés en participar en el estudio. 


\section{Referencias}

1. Otero W, Gómez M, Castro D. Gastric carcinogenesis. Revista Colombiana de Gastroenterología. 2009;24:314-29.

2. IARC Helicobacter pylori Working Group. Helicobacter pylori eradication as a strategy for preventing gastric cancer. Lyon: International Agency for Research in Cancer; 2014. p. 1-59.

3. Sugano K, Tack J, Kuipers EJ, Graham DY, El-Omar EM, Miura S, et al. Kyoto global consensus report on Helicobacter pylori gastritis. Gut. 2015;64:1-15. https://doi.org/10.1136/gutjnl-2015-309252

4. Malfertheiner P, Megraud F, Morain CAO, Gisbert JP, Kuipers EJ, Axon AT, et al. Management of Helicobacter pylori infection-the Maastricht V/Florence Consensus Report. Gut. 2017;66:6-30. https://doi.org/10.1136/gutjnl-2016-312288

5. Savoldi A, Carrara E, Graham DY, Conti M, Tacconelli E. Prevalence of antibiotic resistance in Helicobacter pylori: A systematic review and meta-analysis in World Health Organization Regions. Gastroenterology. 2018 Gastroenterol. 2018;155:1372-82. https://doi.org/10.1053/j.gastro.2018.07.007

6. Zhang M. High antibiotic resistance rate: A difficult issue for Helicobacter pylori eradication treatment. World J Gastroenterol. 2015;21:13432-7. https://doi.org/10.3748/wjg.v21.i48.13432

7. Organización Mundial de la Salud. La OMS publica la lista de las bacterias para las que se necesitan urgentemente nuevos antibióticos. Fecha de consulta: 13 de octubre de 2018. Disponible en: http://www.who.int/es/news-room/detail/27-02-2017-who-publishes-list-ofbacteria-for-which-new-antibiotics-are-urgently-needed

8. Hu Y, Zhang M, Lu B, Dai J. Helicobacter pylori and antibiotic resistance, a continuing and intractable problem. Helicobacter. 2016;21:349-63. https://doi.org/10.1111/hel.12299

9. Wueppenhorst N, Stueger HP, Kist M, Glocker E. Identification and molecular characterization of triple- and quadruple-resistant Helicobacter pylori clinical isolates in Germany. J Antimicrob Chemother. 2009;63:648-53. https://doi.org/10.1093/jac/dkp003

10. Otth L, Wilson M, Fernández H, Otth C, Toledo C, Cárcamo V, et al. Isolation of Helicobacter pylori in gastric mucosa and susceptibility to five antimicrobial drugs in southern Chile. Braz J Microbiol. 2011;42:442-7. https://doi.org/10.1590/S1517-83822011000200005

11. De Francesco V, Giorgio F, lerardi E, Zotti M, Neri M, Milano A, et al. Primary clarithromycin resistance in Helicobacter pylori: The multicentric Italian clarithromycin resistance observational (MICRO) study. J Gastrointestin Liver Dis. 2011;20:235-9.

12. Gerrits MM, Schuijffel D, van Zwet AA, Kuipers EJ, Vandenbroucke-Grauls CM, Kusters JG. Alterations in penicillin-binding protein $1 \mathrm{~A}$ confer resistance to beta-lactam antibiotics in Helicobacter pylori. Antimicrob Agents Chemother. 2002;46:2229-33. https://doi.org/10.1128\%2FAAC.46.7.2229-2233.2002

13. Rimbara E, Noguchi N, Kawai T, Sasatsu M. Mutations in penicillin-binding proteins 1,2 and 3 are responsible for amoxicillin resistance in Helicobacter pylori. J Antimicrob Chemother. 2008;61:995-8. https://doi.org/10.1093/jac/dkn051

14. Trespalacios AA, Otero W, Caminos JE, Mercado MM, Ávila J, Rosero LE, et al. Phenotypic and genotypic analysis of clarithromycin-resistant Helicobacter pylori from Bogotá D.C., Colombia. J Microbiol. 2013;51:448-52. https://doi.org/10.1007/s12275-013-2465-6

15. Kwon DH, Peña JA, Osato MS, Fox JG, Graham DY, Versalovic J. Frameshift mutations in rdxA and metronidazole resistance in North American Helicobacter pylori isolates. $J$ Antimicrob Chemother. 2000;46:793-6.

16. Gerrits MM, Zoete MR De, Arents NL, Kuipers EJ, Kusters JG. 16S rRNA mutation-mediated tetracycline resistance in Helicobacter pylori. Antimicrob Agents Chemother. 2002;46:29963000. https://doi.org/10.1128/AAC.46.9.2996-3000.2002

17. Zhang Z, Liu Z, Zheng P, Tang F, Yang P. Influence of efflux pump inhibitors on the multidrug resistance of Helicobacter pylori. World J Gastrointerol. 2010;16:1279-84. https://doi.org/10.3748/wjg.v16.i10.1279

18. Kutschke A, de Jonge BLM. Compound efflux in Helicobacter pylori compound efflux in Helicobacter pylori. Antimicrob Agents Chemother. 2005;49:3009-11. https://doi.org/10.1128/AAC.49.7.3009-3010.2005

19. Falsafi T, Ehsani A, Attaran B, Niknam V. Association of hp1181 and hp1184 genes with the active efflux phenotype in multidrug-resistant isolates of Helicobacter pylori. Jundishapur $\mathrm{J}$ Microbiol. 2016;20:e30726. https://doi.org/10.5812/jjm.30726 
20. Liu Z, Zheng P, Yang P. Efflux pump gene hefA of Helicobacter pylori plays an important role in multidrug resistance. World J Gastroenterol. 2008;14:5217-22. https://doi.org/10.3748/wjg.14.5217

21. Trespalacios AA, Otero Regino W, Mercado Reyes M. Helicobacter pylori resistance to metronidazole, clarithromycin and amoxicillin in Colombian patients. Revista Colombiana de Gastroenterología. 2010;25:31-8.

22. Trespalacios AA, Otero W, Arévalo GA, Poutou RA, Rimbara E, Graham DY. Surveillance of levofloxacin resistance in Helicobacter pylori isolates in Bogotá-Colombia (2009-2014). PLoS ONE. 2016;11:1-10. https://doi.org/10.1371/journal.pone.0160007.

23. Heep M, Kist M, Strobel S, Beck D, Lehn N. Secondary resistance among 554 isolates of Helicobacter pylori after failure of therapy. Eur J Clin Microbiol Infect Dis. 2000;19:538-41.

24. Boltin D, Ben-Zvi H, Perets TT, Kamenetsky Z, Samra Z, Dickman R, et al. Trends in secondary antibiotic resistance of Helicobacter pylori from 2007 to 2014: Has the tide turned? J Clin Microbiol. 2015;53:522-7. https://doi.org/10.1128/JCM.03001-14

25. Rugge M, Correa P, Di Mario F, El-Omar E, Fiocca R, Geboes K, et al. OLGA staging for gastritis: A tutorial. Dig Liver Dis. 2008;40:650-8. https://doi.org/10.1016/j.dld.2008.02.030

26. Patel JB, Cockerill III FR, Alder J, Bradford PA, Eliopoulos GM, Hardy DJ, et al. Performance standards for antimicrobial susceptibility testing; Twenty-Fourth Informational Supplement. Wayne: CLSI; 2014. p. 1-230.

27. Wikler MA, Bush K, Cockerill III FR, Dudley MN, Eliopoulos GM, Hardy DJ, et al. Performance standards for antimicrobial susceptibility testing; Eighteenth Informational Supplement. Wayne: CLSI; 2008. p. 1-188.

28. Mégraud F. Antibiotic resistance in Helicobacter pylori infection. Br J Bull. 1998;54:207-16.

29. Mégraud F, Bénéjat L, Nina E, Ngoyi O, Lehours P. Molecular approaches to identify Helicobacter pylori antimicrobial resistance. Gastroenterol Clin North Am. 2015;44:577-96. https://doi.org/10.1016/j.gtc.2015.05.002

30. Mégraud F, Lehours P. Helicobacter pylori detection and antimicrobial susceptibility testing Clin Microbiol Rev. 2007;20:280-322. https://doi.org/10.1128/CMR.00033-06

31. Glocker E, Stueger H, Kist M. Quinolone resistance in Helicobacter pylori isolates in Germany. Antimicrob Agents Chemother. 2007;51:346-9. https://doi.org/10.1128/AAC.00614-06

32. Kim KS, Kang JO, Eun CS, Han DS, Choi TY. Mutations in the 23S rRNA gene of Helicobacter pylori associated with clarithromycin resistance. J Korean Med Sci. 2002;17:599-603. https://doi.org/10.3346/jkms.2002.17.5.599

33. Tankovic J, Lascols C, Sculo Q, Petit J. Single and double mutations in gyrA but not in gyrB are associated with low- and high-level fluoroquinolone resistance in Helicobacter pylori. Antimicrob Agents Chemother. 2003;47:3942-4. https://doi.org/10.1128/AAC.47.12.3942-3944.2003

34. Ogata SK, Godoy APO, da Silva Patricio FR, Kawakami E. High Helicobacter pylori resistance to metronidazole and clarithromycin in Brazilian children and adolescents. J Pediatr Gastroenterol Nutr. 2013;56:645-8. https://doi.org/10.1097/MPG.0b013e31828b3669

35. Mégraud F. Current recommendations for Helicobacter pylori therapies in a world of evolving resistance. Gut Microbes. 2013;4:541-8. https://doi.org/10.4161/gmic.25930

36. Boyanova L. Prevalence of multidrug-resistant Helicobacter pylori in Bulgaria. J Med Microbiol. 2009;58:930-5. https://doi.org/10.1099/jmm.0.009993-0

37. Karczewska E, Wojtas-bonior I, Sito E, Budak A. Primary and secondary clarithromycin, metronidazole, amoxicillin and levofloxacin resistance to Helicobacter pylori in southern Poland. Pharmacol Rep. 2011;63:799-807.

38. Park CS, Lee SM, Park CH, Koh HR, Jun CH, Park SY, et al.Pretreatment antimicrobial susceptibility-guided vs. clarithromycin-based triple therapy for Helicobacter pylori eradication in a region with high rates of multiple drug resistance. Am J Gastroenterol. 2014:109:1595-602. https://doi.org/10.1038/ajg.2014.222

39. Selgrad M, Tammer I, Langner C, Bornschein J, Meißle J, Kandulski A, et al. Different antibiotic susceptibility between antrum and corpus of the stomach, a possible reason for treatment failure of Helicobacter pylori infection. World J Gastroenterol. 2014;20:16245-51. https://doi.org/10.3748/wjg.v20.i43.16245

40. Yakoob J, Fan X, Hu G, Yang H, Liu L, Liu S, et al. Polycolonization of Helicobacter pylori among Chinese subjects. Clin Microbiol Infect. 2001;7:187-92. https://doi.org/10.1046/j.1198-743x.2001.00226.x 
41. Kim JJ, Kim JG, Kwon DH. Mixed-infection of antibiotic susceptible and resistant Helicobacter pylori isolates in a single patient and underestimation of antimicrobial susceptibility testing. Helicobacter. 2003;8:202-6. https://doi.org/10.1046/j.1523-5378.2003.00145.x

42. Otero W, Gómez M, Otero L,Trespalacios A. Helicobacter pylori: ¿cómo se trata en el 2018? Rev Gastroenterol Peru. 2018;38:54-63.

43. Otero W, Trespalacios AA, Otero L, Vallejo M, Torres M, Pardo R, et al. Guía práctica para el diagnóstico y tratamiento de la infección por Helicobacter pylori en adultos. Revista Colombiaba de Gastroenterología. 2015;30(Suppl.1):17-33.

44. Graham DY, Lee YC, Wu MS. Rational Helicobacter pylori therapy: Evidence-based medicine rather than medicine-based evidence. Clin Gastroenterol Hepatol. 2014;12:17786. https://doi.org/10.1016/j.cgh.2013.05.028

45. Wu IT, Chuah SK, Lee CH, Liang CM, Lu LS, Kuo YH, et al. Five-year sequential changes in secondary antibiotic resistance of Helicobacter pylori in Taiwan. World J Gastroenterol. 2015;21:10669-74. https://doi.org/10.3748/wjg.v21.i37.10669

46. Boyanova L, Evstatiev I, Gergova G, Yaneva P, Mitov I. Linezolid susceptibility in Helicobacter pylori, including strains with multidrug resistance. Int J Antimicrob Agents. 2015;46:703-6. https://doi.org/10.1016/j.ijantimicag.2015.08.010

47. Shi J, Jiang $\mathrm{Y}$, Zhao $\mathrm{Y}$. Promising in vitro and in vivo inhibition of multidrug-resistant Helicobacter pylori by linezolid and novel oxazolidinone analogues. J Glob Antimicrob Resist. 2016;7:106-9. https://doi.org/10.1016/j.jgar.2016.07.016 